\title{
Double real radiation corrections to top-antitop production at the LHC
}

\author{
Gabriel Abelof* \\ ETH Zürich \\ E-mail: abelof@itp.phys.ethz.ch
}

\section{Aude Gehrmann-De Ridder}

ETH Zürich

E-mail: gehra@itp.phys.ethz.ch

\begin{abstract}
We employ the antenna subtraction method to construct subtraction terms for certain partonic processes contributing to the double real radiation corrections to $t \bar{t}$ production. These subtraction terms are calculated for both leading and subleading colour contributions and they require the use of new four-parton antenna functions with massive fermions. The validity of our subtraction terms is then checked numerically: We show that in all the unresolved regions of phase space the ratio of the double real radiation contributions and the subtraction terms approaches unity.
\end{abstract}

"Loops and Legs in Quantum Field Theory" 11th DESY Workshop on Elementary Particle Physics April 15-20, 2012

Wernigerode, Germany

${ }^{*}$ Speaker. 


\section{Introduction}

With a mass $m_{t}=173 \pm 1.3 \mathrm{GeV}$, the top quark is the heaviest Standard Model particle that is produced at colliders. The detailed study of its properties will shed light on the origin of particle masses and the mechanism of electroweak symmetry breaking. Since its discovery at the Fermilab Tevatron [1,2], a number of these properties (mass, couplings) have been determined to an accuracy of ten to twenty per cent. The large number of top quark pairs produced at the LHC [3] will enable more precise experimental measurements. Some observables such as the total cross section for top quark pair production will be determined with an accuracy of the order of five per cent. Therefore, in order to extract fundamental parameters like couplings and masses by comparing experimental data and theoretical predictions, the latter should also be computed to an accuracy of a few percent. This is achieved by evaluating higher order terms in the perturbative expansion of the top-antitop cross section.

The next-to-leading-order (NLO) predictions for top quark pair production in the narrow width approximation have been available for quite some time [4], and more recently off-shell effects have been taken into account in [5]. Combined with the next-to-leading-logarithmic resummation (NLL) [6] and the NNLL resummation [7], these calculations yield a theoretical uncertainty of the order of ten percent. The same accuracy is available for single top production [8], top pair in association with jets [9] and for top-pair-plus-bottom-pair production [10].

An increasing number of pieces relevant for the evaluation of the cross section for top pair production at NNLO have become available recently. Most notably, the full NNLO corrections to the quark-antiquark channel have been computed in [11]. Quite generally, the NNLO corrections to any given partonic cross section with $m$ particles in the final state contain three different contributions

$$
\mathrm{d} \hat{\sigma}_{N N L O}=\int_{\mathrm{d} \Phi_{m+2}} \mathrm{~d} \hat{\sigma}_{N N L O}^{R R}+\int_{\mathrm{d} \Phi_{m+1}} \mathrm{~d} \hat{\sigma}_{N N L O}^{R V}+\int_{\mathrm{d} \Phi_{m}} \mathrm{~d} \hat{\sigma}_{N N L O}^{V V} .
$$

The double virtual corrections $\mathrm{d} \hat{\sigma}_{N N L O}^{V V}$ are constructed with two-loop amplitudes interfered with the corresponding Born matrix element, and with one-loop amplitudes squared. The mixed real-virtual contributions d $\hat{\sigma}_{N N L O}^{R V}$ are built from the interference of one-loop amplitudes with an additional real radiated parton and the corresponding tree-level matrix element. Finally, the double real radiation corrections d $\hat{\sigma}_{N N L O}^{R R}$ are constructed from tree-level matrix elements squared with two real partons added to the corresponding basic LO process.

For top quark pair production in hadronic collisions, intermediate results are available in all three NNLO ingredients with a varying degree of completion. The double virtual corrections built with one-loop amplitudes squared have been fully worked out in [12]. Regarding the two-loop matrix elements, there is a purely numerical evaluation of the $q \bar{q} \rightarrow t \bar{t}$ channel [13]. There are also analytical results for several colour factors in both quark and gluon-initiated processes [14]. For the mixed real-virtual contributions, the infrared structure of the corresponding one-loop matrix elements has been studied in [15]. The double real radiation corrections have been tackled with two independent approaches: On one hand, there is a numerical approach that combines the techniques of sector decomposition and subtraction [16], and on the other hand the antenna subtraction method with massive fermions has been derived and employed by ourselves in [17, 18, 19, 20, 21]. Here we shall review how we apply this method to the partonic processes 


$$
q \bar{q} \rightarrow t \bar{t} q^{\prime} \bar{q}^{\prime}, \quad q \bar{q} \rightarrow t \bar{t} q \bar{q}, \quad q q^{\prime} \rightarrow t \bar{t} q q^{\prime}, \quad q q \rightarrow t \bar{t} q q, \quad g g \rightarrow t \bar{t} q \bar{q} .
$$

In section 2 we outline the way in which this extended version of the antenna subtraction method works at NNLO. The necessary ingredients required for the construction of subtraction terms for the double real radiation corrections to heavy quark pair production in hadronic collisions are reviewed in section 3. In section 4 we present the results of a series of numerical checks carried out in order to test the validity of our subtraction terms. Finally, in section 5 we present our conclusions.

\section{Antenna subtraction at NNLO}

While infrared singularities from purely virtual corrections are obtained immediately after integration over the loop momenta, their extraction is more involved for real emission (or mixed real-virtual) contributions. There, the infrared singularities only become explicit after integrating the matrix elements over the phase space appropriate to the differential cross section under consideration. Since these observables depend in general in a non trivial manner on the experimental criteria needed to define them, they can only be calculated numerically. The computation of hadronic observables including higher order corrections therefore requires a systematic procedure to cancel infrared singularities among different partonic channels before any numerical computation of the observable can be performed. Subtraction methods explicitly constructing infrared subtraction terms are well-known solutions to this problem.

Employing a subtraction method, the NNLO partonic cross section given in 1.1 for a hadronic observable can be organised as

$$
\begin{aligned}
\mathrm{d} \hat{\sigma}_{N N L O} & =\int_{\mathrm{d} \Phi_{m+2}}\left(\mathrm{~d} \hat{\sigma}_{N N L O}^{R R}-\mathrm{d} \hat{\sigma}_{N N L O}^{S}\right) \\
& +\int_{\mathrm{d} \Phi_{m+1}}\left(\mathrm{~d} \hat{\sigma}_{N N L O}^{R V}-\mathrm{d} \hat{\sigma}_{N N L O}^{V S}+\mathrm{d} \hat{\sigma}_{N N L O}^{M F, 1}\right) \\
& +\int_{\mathrm{d} \Phi_{m}}\left(\mathrm{~d} \hat{\sigma}_{N N L O}^{V V}+\int_{2} \mathrm{~d} \hat{\sigma}_{N N L O}^{S}+\int_{1} \mathrm{~d} \hat{\sigma}_{N N L O}^{V S}+\mathrm{d} \hat{\sigma}_{N N L O}^{M F, 2}\right) .
\end{aligned}
$$

The crucial points that all subtraction terms $\mathrm{d} \hat{\sigma}_{N N L O}^{S}$ and $\mathrm{d} \hat{\sigma}_{N N L O}^{V S}$ must satisfy are that (a) they approximate $\mathrm{d} \hat{\sigma}_{N N L O}^{R R}$ and $\mathrm{d} \hat{\sigma}_{N N L O}^{R V}$ respectively in all singular limits, (b) they are still sufficiently simple to be integrated analytically, (c) they should account for the limit they are aimed at without introducing spurious infrared singularities in other limits. With these requirements, the integrand in each of the three lines of eq.(2.1) is free of infrared singularities, and the corresponding phase space integrals can be carried out numerically in four dimensions.

Based on the universal factorisation of QCD amplitudes in their infrared limits [22], the antenna subtraction method provides a framework to construct subtraction terms meeting the requirements explained above. It was first developed for the evaluation of NNLO jet rates in $e^{+} e^{-}$ collisions [23, 24], and then extended in order to deal with coloured initial states and massless final state partons $[25,26]$. The formalism for the construction of NNLO antenna subtraction terms for hadronic jet observables has been set up in [27, 28] in the context of a proof-of-principle implementation of the contribution of the purely gluonic contributions to di-jet production at hadron colliders. 
The method has also been expanded to include massive fermions. In $[17,18]$ it was extended at the NLO level and employed to compute the real NLO corrections to $t \bar{t}$ and $t \bar{t}+$ jet hadronic production. In $[19,20]$ we further extended the method to the NNLO level, and derived the double real emission corrections to the cross section for $t \bar{t}$ production due to the partonic channels mentioned above.

The main ingredients for the construction of antenna subtraction terms are antenna functions, phase space mappings, and a Lorentz invariant factorisation of the full phase space.

Antenna functions are calculated as ratios of physical colour-ordered matrix elements squared with two hard particles (hard radiators) and unresolved radiation emitted in between. They yield the correct unresolved factor (splitting functions, soft factors, etc.) corresponding to each limit. In the evaluation of the double real radiation only tree-level antennae are needed. The subtraction of single unresolved limits requires three-parton antennae which are calculated as the ratio of a three-parton and a two-parton colour-ordered matrix element squared

$$
X_{i j k}^{0}=S_{i j k, I K} \frac{\left|\mathscr{M}_{i j k}^{0}\right|^{2}}{\left|\mathscr{M}_{I K}^{0}\right|^{2}} .
$$

To subtract double unresolved limits we employ products of two three-parton antennae or four parton antennae depending on the colour connection of the unresolved particles. Four-parton antenna are defined as

$$
X_{i j k l}^{0}=S_{i j k l, I L} \frac{\left|\mathscr{M}_{i j k l}^{0}\right|^{2}}{\left|\mathscr{M}_{I L}^{0}\right|^{2}} .
$$

We distinguish between final-final (f-f), initial-final (i-f) and initial-initial (i-i) antennae depending on whether the hard radiators are in the final or in the initial state. Furthermore, since the hard radiators can be (anti)quarks or gluons we distinguish between quark-antiquark, quark-gluon, and gluon-gluon antennae.

Subtraction terms are then built as products of antenna functions and reduced matrix elements with remapped momenta. Although we shall leave the phase-space mappings to be discussed elsewhere (e.g. [19, 27]), their main feature is that they collapse onto the Born kinematics of each unresolved limit. Different mappings are needed for $\mathrm{f}-\mathrm{f}$, i-f and i-i configurations.

The last ingredient needed to construct antenna subtraction terms is a factorisation of the full double real radiation phase space into an antenna phase space and a reduced one with remapped momenta. This factorisation, which is different for $\mathrm{f}-\mathrm{f}, \mathrm{i}-\mathrm{f}$ and $\mathrm{i}-\mathrm{i}$ configurations, enables the analytic integration of the antenna functions in the subtraction terms over the antenna phase space, leaving the reduced matrix elements with remapped momenta unintegrated. Schematically, the integrated form of a subtraction term built with a four-parton antenna reads

$$
\mathscr{X}_{i j k l}^{0} \int \mathrm{d} \Phi_{m}\left|\mathscr{M}_{m+2}\right|^{2}
$$

with $\mathscr{X}_{i j k l}^{0}$ being the integrated antenna, $\mathrm{d} \Phi_{m}$ the reduced phase space and $\left|\mathscr{M}_{m+2}\right|^{2}$ the reduced matrix element squared. This integrated subtraction term can then be readily combined with the double virtual contributions and with the corresponding mass factorisation counterterms, thus achieving an analytic cancellation of the explicit poles in the dimensional regulator $\varepsilon$. 


\section{Subtraction terms for $t \bar{t}$ production in hadronic collisions}

In the partonic processes

$$
q \bar{q} \rightarrow t \bar{t} q^{\prime} \bar{q}^{\prime}, \quad q \bar{q} \rightarrow t \bar{t} q \bar{q}, \quad q q^{\prime} \rightarrow t \bar{t} q q^{\prime}, \quad q q \rightarrow t \bar{t} q q, \quad g g \rightarrow t \bar{t} q \bar{q}
$$

contributing to the double real corrections to $t \bar{t}$ production in hadronic collisions we encounter

- Soft quark-antiquark limits

- Initial-final triple collinear limits

- Double initial-final collinear limits

- Single final-final and initial-final collinear limits.

There are no collinear limits involving the heavy quark pair, since those are regulated by the large fermion mass. The quasi-collinear $\operatorname{logarithms} \log \left(m_{t}^{2} / s_{i j}\right)$, which could be a source of numerical instabilities for example in bottom-antibottom production, are not enhanced in the kinematical configuration under consideration. These limits are therefore not taken into account in the construction of our subtraction terms.

The subtraction of the infrared limits listed above requires several kinds of three-parton treelevel antenna functions with massive and massless hard radiators in i-i, i-f and f-f configurations. It also requires the massless (i-i) four-parton antennae $B_{4}^{0}\left(\hat{\bar{q}}, \bar{q}^{\prime}, q^{\prime}, \hat{q}\right)$ and $G_{4}^{0}\left(\hat{g}, q^{\prime}, \bar{q}^{\prime}, \hat{g}\right)$, the massive (i-f) antennae $B_{4}^{0}\left(Q, \bar{q}^{\prime}, q^{\prime}, \hat{q}\right), E_{4}^{0}\left(Q, \bar{q}^{\prime}, q^{\prime}, \hat{g}\right)$ and $\tilde{E}_{4}^{0}\left(Q, \bar{q}^{\prime}, q^{\prime}, \hat{g}\right)$ and the massive (f-f) antenna $B_{4}^{0}\left(Q, \bar{q}^{\prime}, q^{\prime}, \bar{Q}\right)$. These four-parton antennae are employed to subtract those infrared limits of the double real radiation matrix element in which the two unresolved particles are colour-connected.

The massless (i-i) antennae are simply obtained by the appropriate crossings of their massless counterparts in [24]. Their integrated form has been calculated in [26]. The massive (f-f) B-type antenna has been computed for the first time and integrated in [21]. The unintegrated form of the massive (i-f) B and E type antennae has been presented more recently in $[19,20]$. Their integration is ongoing [29].

While the subtraction of double unresolved limits in the leading colour pieces follows the general antenna subtraction framework, certain subleading colour terms require a special treatment. In the leading colour contributions the subtraction of a double unresolved limit is always achieved with only one four-parton antenna (and products of three-parton antennae), whereas for some types of subleading colour pieces, subtraction terms must be built with a combination of several fourparton antennae. The particular combination of antenna functions that are needed is obtained by analysing the infrared behaviour of the matrix element at the amplitude level [19,20].

\section{Numerical results}

To verify how well the subtraction terms approximate the double real contributions related to the different partonic processes that we considered, we have used RAMBO [30] to generate phase space points in the vicinity of the singular regions and computed the ratio

$$
R=\frac{\mathrm{d} \hat{\sigma}_{N N L O}^{R R}}{\mathrm{~d} \hat{\sigma}_{N N L O}^{S}}
$$


for each of these points. In each unresolved limit we define a control variable $x$ that allows us to vary the proximity of the phase space points to the singularity. For the difference $\mathrm{d} \hat{\sigma}_{N N L O}^{R R}-\mathrm{d} \hat{\sigma}_{N N L O}^{S}$ to be finite and numerically integrable in four dimensions, the ratio $R$ should approach unity as we get close to any singularity. The phase space points were generated with a fixed centre-of-mass energy of $\sqrt{s}=1000 \mathrm{GeV}$, the heavy fermions were given a mass of $174.3 \mathrm{GeV}$, and required to have $p_{T}>50 \mathrm{GeV}$.

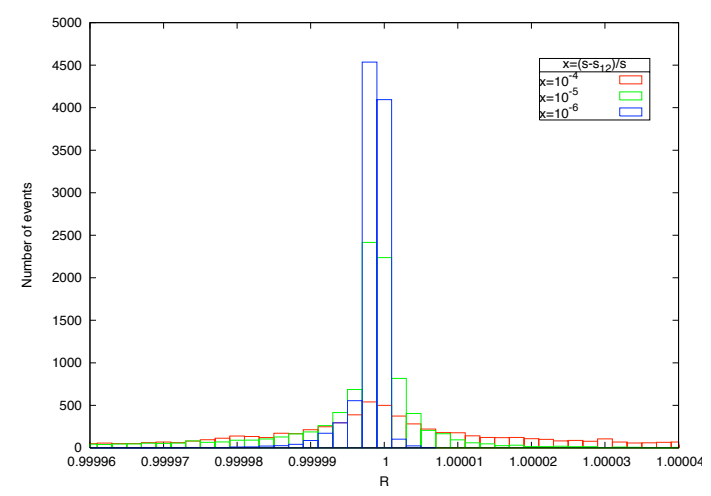

(a)

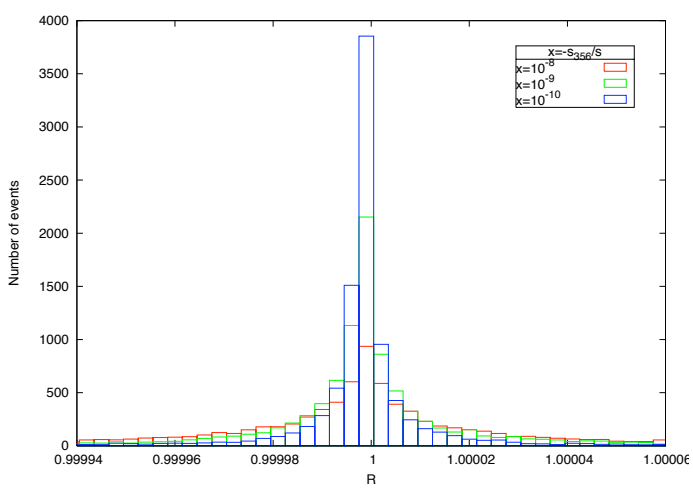

(b)

Figure 1: Distribution of $\mathrm{R}$ for 10000 phase space points in (a) the double soft region and (b) the triple collinear region, in the partonic process $g g \rightarrow Q \bar{Q} q \bar{q}$.

In fig. 1 we show the ratio between the double real radiation matrix element and the subtraction for (a) the double soft and (b) the triple collinear region of the process $g g \rightarrow Q \bar{Q} q \bar{q}$. In the former we use $x=\left(s-s_{Q \bar{Q}}\right) / s$ as our control variable, while in the later we employ $x=-s_{\hat{g} q \bar{q}} / s$. As it can be seen as $x$ becomes smaller, i.e. as we get closer in phase space to the singularity, the histograms become more sharply peaked at $R=1$. The same behaviour is observed for all single and double unresolved limits of the partonic processes considered. This is a clear signal that our subtraction terms correctly approximate the real radiation matrix elements in all unresolved regions of phase space.

As explained in [19], for example, antenna subtraction terms do not reproduce the spin correlations of the gluon collinear splittings. However, as it has been shown in $[19,27]$, these spin correlations are proportional to $\cos (2 \phi+\alpha)$, where $\phi$ is the azimuthal angle of the collinear pair about the collinear axis. The spin correlations can therefore be averaged out by combining phase space points whose azimuthal angles about the collinear axis differ by $\pi / 2$. As it can be seen in fig.2, this combination indeed leads to a remarkable improvement in the convergence of our subtraction terms to the real radiation matrix element.

\section{Conclusions}

We generalised the antenna subtraction method to evaluate the double real radiation corrections to heavy quark pair production in hadronic collisions. We applied this method to the construction of subtraction terms for the partonic processes

$$
q \bar{q} \rightarrow t \bar{t} q^{\prime} \bar{q}^{\prime}, \quad q \bar{q} \rightarrow t \bar{t} q \bar{q}, \quad q q^{\prime} \rightarrow t \bar{t} q q^{\prime}, \quad q q \rightarrow t \bar{t} q q, \quad g g \rightarrow t \bar{t} q \bar{q} .
$$




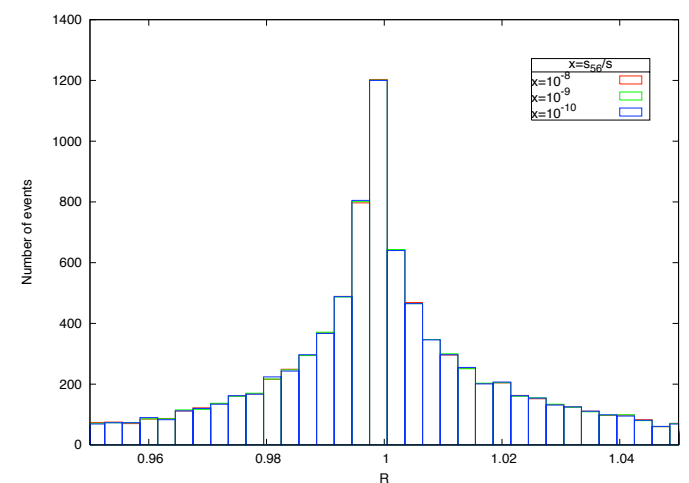

(a)

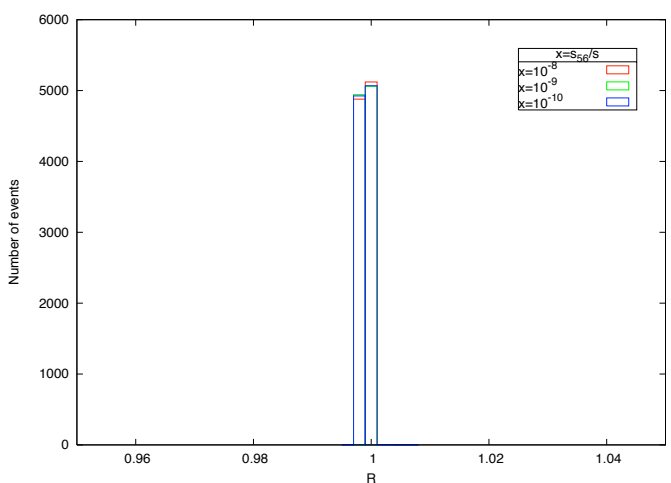

(b)

Figure 2: Distribution of $\mathrm{R}$ for 10000 phase space points in the collinear region: (a) without azimuthal averaging, (b) with azimuthal averaging, in the partonic process $q \bar{q} \rightarrow Q \bar{Q} q^{\prime} \bar{q}^{\prime}$.

For this purpose, we computed the new relevant massive initial-final antenna functions and their corresponding infrared limits. The integration of them is ongoing. As a crucial test of our subtraction terms we checked numerically that they correctly approximate the double real radiation matrix elements in all their unresolved limits. This constitutes a highly nontrivial check on our extension of the antenna subtraction method to treat massive final states at NNLO.

The computation of the double real corrections and the corresponding subtraction terms for the partonic processes mentioned above represents an essential step towards the completion of a full NNLO calculation for $t \bar{t}$ production in hadronic collisions.

\section{Acknowledgements}

This research was supported by the Swiss National Science Foundation (SNF) under contract PP00P2-139192 and in part by the European Commission through the 'LHCPhenoNet' Initial Training Network PITN-GA-2010-264564', which are hereby acknowledged.

\section{References}

[1] F. Abe et al. [CDF Collaboration], Observation of top quark production in $\bar{p} p$ collisions, Phys. Rev. Lett. 74, 2626 (1995).

[2] S. Abachi et al. [D0 Collaboration], Observation of the top quark, Phys. Rev. Lett. 74, 2632 (1995).

[3] P. Silva et al. [ATLAS and CMS Collaborations], "Recent results on Top quark Physics with the ATLAS and CMS experiments," arXiv:1206.2967 [hep-ex].

[4] P. Nason, S. Dawson and R. K. Ellis, The One Particle Inclusive Differential Cross-Section for Heavy Quark Production in Hadronic Collisions, Nucl. Phys. B327, 49 (1989) [Erratum-ibid. B335, 260 (1990)]; W. Beenakker, W. L. van Neerven, R. Meng, G. A. Schuler and J. Smith, QCD corrections to heavy quark production in hadron hadron collisions, Nucl. Phys. B351, 507 (1991).

[5] A. Denner, S. Dittmaier, S. Kallweit and S. Pozzorini, NLO QCD corrections to WWbb production at hadron colliders, Phys. Rev. Lett. 106, 052001 (2011); G. Bevilacqua, M. Czakon, A. van Hameren, C. G. Papadopoulos and M. Worek, Complete off-shell effects in top quark pair hadroproduction with leptonic decay at next-to-leading order, JHEP 1102, 083 (2011). 
[6] S. Moch and P. Uwer, Theoretical status and prospects for top-quark pair production at hadron colliders, Phys. Rev. D78, 034003 (2008); M. Cacciari, S. Frixione, M. L. Mangano, P. Nason and G. Ridolfi, Updated predictions for the total production cross sections of top and of heavier quark pairs at the Tevatron and at the LHC, JHEP 0809, 127 (2008); N. Kidonakis and R. Vogt, The Theoretical top quark cross section at the Tevatron and the LHC, Phys. Rev. D78, 074005 (2008).

[7] V. Ahrens, A. Ferroglia, M. Neubert, B. D. Pecjak and L. L. Yang, Renormalization-Group Improved Predictions for Top-Quark Pair Production at Hadron Colliders, JHEP 1009, 097 (2010).

[8] B. W. Harris, E. Laenen, L. Phaf, Z. Sullivan and S. Weinzierl, The Fully differential single top quark cross-section in next to leading order QCD, Phys. Rev. D66, 054024 (2002).

[9] S. Dittmaier, P. Uwer and S. Weinzierl, NLO QCD corrections to t anti-t + jet production at hadron colliders, Phys. Rev. Lett. 98, 262002 (2007); K. Melnikov and M. Schulze, NLO QCD corrections to top quark pair production in association with one hard jet at hadron colliders, Nucl. Phys. B840, 129 (2010); G. Bevilacqua, M. Czakon, C. G. Papadopoulos and M. Worek, Dominant QCD Backgrounds in Higgs Boson Analyses at the LHC: A Study of pp $\rightarrow$ t anti- $t+2$ jets at Next-To-Leading Order, Phys. Rev. Lett. 104, 162002 (2010); A. Denner, S. Dittmaier, S. Kallweit and S. Pozzorini, arXiv:1208.4053 [hep-ph]; K. Melnikov, A. Scharf and M. Schulze, Phys. Rev. D 85, 054002 (2012) [arXiv:1111.4991 [hep-ph]].

[10] G. Bevilacqua, M. Czakon, C. G. Papadopoulos, R. Pittau and M. Worek, “Assault on the NLO Wishlist: pp -> tt bb, JHEP 0909, 109 (2009); A. Bredenstein, A. Denner, S. Dittmaier and S. Pozzorini, NLO QCD corrections to top anti-top bottom anti-bottom production at the LHC: 2. full hadronic results, JHEP 1003, 021 (2010).

[11] P. Baernreuther, M. Czakon and A. Mitov, "Percent level precision physics at the Tevatron: first genuine NNLO QCD corrections to q qbar -> t tbar + X," arXiv:1204.5201 [hep-ph]; M. Czakon and A. Mitov, "NNLO corrections to top-pair production at hadron colliders: the all-fermionic scattering channels," arXiv:1207.0236 [hep-ph].

[12] J. G. Korner, Z. Merebashvili and M. Rogal, “NNLO $O\left(\alpha(s)^{(4)}\right.$ results for heavy quark pair production in quark-antiquark collisions: The One-loop squared contributions, Phys. Rev. D77, 094011 (2008); C. Anastasiou and S. M. Aybat, The one-loop gluon amplitude for heavy-quark production at NNLO, Phys. Rev. D78, 114006 (2008); B. Kniehl, Z. Merebashvili, J. G. Korner and M. Rogal, Heavy quark pair production in gluon fusion at next-to-next-to-leading $O\left(\alpha_{s}^{(4)}\right)$ order: One-loop, Phys. Rev. D78, 094013 (2008).

[13] M. Czakon, Tops from Light Quarks: Full Mass Dependence at Two-Loops in QCD, Phys. Lett. B664, 307 (2008).

[14] R. Bonciani, A. Ferroglia, T. Gehrmann, D. Maitre and C. Studerus, Two-Loop Fermionic Corrections to Heavy-Quark Pair Production: The Quark-Antiquark Channel, JHEP 0807, 129 (2008);

R. Bonciani, A. Ferroglia, T. Gehrmann and C. Studerus, Two-Loop Planar Corrections to Heavy-Quark Pair Production in the Quark-Antiquark Channel, JHEP 0908, 067 (2009); R. Bonciani, A. Ferroglia, T. Gehrmann, A. Manteuffel and C. Studerus, Two-Loop Leading Color Corrections to Heavy-Quark Pair Production in the Gluon Fusion Channel, JHEP 1101, 102 (2011).

[15] I. Bierenbaum, M. Czakon and A. Mitov, The singular behavior of one-loop massive QCD amplitudes with one external soft gluon, arXiv:1107.4384 [hep-ph].

[16] M. Czakon, Double-real radiation in hadronic top quark pair production as a proof of a certain concept, Nucl. Phys. B849, 250 (2011). 
[17] A. Gehrmann-De Ridder and M. Ritzmann, "NLO Antenna Subtraction with Massive Fermions," JHEP 0907, 041 (2009) [arXiv:0904.3297 [hep-ph]]

[18] G. Abelof and A. Gehrmann-De Ridder, "Antenna subtraction for the production of heavy particles at hadron colliders,” JHEP 1104, 063 (2011) [arXiv:1102.2443 [hep-ph]].

[19] G. Abelof and A. Gehrmann-De Ridder, "Double real radiation corrections to $t \bar{t}$ production at the LHC: the all-fermion processes,” JHEP 1204, 076 (2012) [arXiv:1112.4736 [hep-ph]]

[20] G. Abelof and A. Gehrmann-De Ridder, "Double real radiation corrections to $t \bar{t}$ production at the LHC: the $g g \rightarrow t \bar{t} q \bar{q}$ channel," arXiv:1207.6546 [hep-ph].

[21] W. Bernreuther, C. Bogner and O. Dekkers, "The real radiation antenna function for $S \rightarrow Q \bar{Q} q \bar{q}$ at NNLO QCD,” JHEP 1106, 032 (2011) [arXiv:1105.0530 [hep-ph]];

[22] J. M. Campbell, E. W. N. Glover, Double unresolved approximations to multiparton scattering amplitudes, Nucl. Phys. B527, 264-288 (1998). [hep-ph/9710255]; S. Catani, M. Grazzini, Collinear factorization and splitting functions for next-to-next-to-leading order QCD calculations, Phys. Lett. B446, 143-152 (1999). [hep-ph/9810389]; S. Catani, M. Grazzini, Infrared factorization of tree level QCD amplitudes at the next-to-next-to-leading order and beyond, Nucl. Phys. B570, 287-325 (2000). [hep-ph/9908523].

[23] A. Gehrmann-De Ridder, T. Gehrmann, E. W. N. Glover, G. Heinrich, Infrared structure of $e^{+} e^{-} \rightarrow 3$ jets at NNLO, JHEP 0711, 058 (2007).

[24] A. Gehrmann-De Ridder, T. Gehrmann, E. W. N. Glover, Antenna subtraction at NNLO, JHEP 0509, 056 (2005).

[25] A. Daleo, T. Gehrmann and D. Maitre, “Antenna subtraction with hadronic initial states," JHEP 0704, 016 (2007) [hep-ph/0612257]; A. Daleo, A. Gehrmann-De Ridder, T. Gehrmann and G. Luisoni, "Antenna subtraction at NNLO with hadronic initial states: initial-final configurations," JHEP 1001, 118 (2010) [arXiv:0912.0374 [hep-ph]]; T. Gehrmann and P. F. Monni, "Antenna subtraction at NNLO with hadronic initial states: real-virtual initial-initial configurations," JHEP 1112, 049 (2011) [arXiv:1107.4037 [hep-ph]].

[26] R. Boughezal, A. Gehrmann-De Ridder and M. Ritzmann, "Antenna subtraction at NNLO with hadronic initial states: double real radiation for initial-initial configurations with two quark flavours," JHEP 1102, 098 (2011) [arXiv:1011.6631 [hep-ph]]; A. Gehrmann-De Ridder, T. Gehrmann and M. Ritzmann, "Antenna subtraction at NNLO with hadronic initial states: double real initial-initial configurations," arXiv:1207.5779 [hep-ph], to appear in JHEP.

[27] E. W. Nigel Glover, J. Pires, Antenna subtraction for gluon scattering at NNLO, JHEP 1006, 096 (2010). [hep-ph/1003.2824].

[28] A. Gehrmann-De Ridder, E. W. N. Glover and J. Pires, "Real-Virtual corrections for gluon scattering at NNLO,” JHEP 1202, 141 (2012) [arXiv:1112.3613 [hep-ph]].

[29] G. Abelof, A. Gehrmann-De Ridder and O. Dekkers, "Antenna subtraction with massive fermions at NNLO: Double real initial-final configurations,” arXiv:1210.5059 [hep-ph].

[30] R. Kleiss, W. J. Stirling and S. D. Ellis, A new Monte Carlo treatment of multiparticle phase space at high-energies Comput. Phys. Commun. 40 (1986) 359. 